\title{
Anxiety and Stroop Performance
}

\author{
Prof. H.K. Chhabra ${ }^{1}$, Ms. Heena Parveen ${ }^{2}$
}

\section{ABSTRACT}

The study probed the effect of cognitive and emotional Stroop on the performance of students from different levels of anxiety. Anxiety is a normal and healthy reaction to perceived danger that triggers a variety of physical, mental and behavioral changes in order to facilitate a speedy response (WHO, 2004). Again, anxiety has also been found to have an important role to play in situations of cognitive conflict. The Stroop effect has often been widely used for assessing conflict (Stroop, 1935; MacLeod, 1991). In the present study, a mixed sample of 89 students from the Government schools of Chandigarh in the age range of 14-18 years (mean age= 15.9 years) was taken, for the purpose. The repeated measures ANOVA and other analysis of the data brought out performance differences with regard to how different groups of anxiety perform during cognitive and emotional Stroop task situations. The study revealed that anxiety significantly interacts with Stroop performance. Further, high anxiety damages emotional Stroop performance more than it damages the cognitive Stroop performance i.e., high anxiety individuals show more reactive control (Braver, 2012). On the other hand, low and moderate anxiety seems to lead to more errors on cognitive Stroop as compared to the emotional Stroop.

Keywords: Anxiety, Stroop, Emotional, Students

Anxiety is a normal and healthy reaction to perceived danger that triggers a variety of physical, mental and behavioral changes in order to facilitate a speedy response (WHO, 2004). It arises out of the perception of uncontrollability and unavoidability of a situation (Ohman, 2000), which may otherwise be manageable with a specific response. Any conflict based input is likely to be influenced by the anxiety of an individual.

Also, researches indicate that anxiety can affect students behaviorally, emotionally, cognitively, and physiologically. The emotional effects of anxiety may include feelings of apprehension, trouble concentrating, and feeling, anticipating danger, irritability and restlessness. The cognitive effects may include thoughts about suspected dangers, such as fear of dying (Huberty, 2009). In this context, anxiety has been found to have an effect on performance, as anxious students seem to be distracted by irrelevant or incidental aspects of task at hand and they have trouble focusing on significant details of that task (e.g. Nadeem et al., 2012).

\footnotetext{
${ }^{1}$ Department of Psychology, Panjab University, Chandigarh.

${ }^{2}$ Junior Research Fellow (UGC), Department of Psychology, Panjab University, Chandigarh. *Corresponding Author

(C) 2015 I H Chhabra, H Parveen; licensee IJIP. This is an Open Access Research distributed under the terms of the Creative Commons Attribution License (http://creativecommons.org/licenses/by/2.0), which permits unrestricted use, distribution, and reproduction in any Medium, provided the original work is properly cited.
} 
The dual mechanisms of control (DMC) model (Braver, 2012) further suggest that cognitive control is achieved through two distinct modes: proactive and reactive. Proactive control is found to be more cognitively taxing of the two modes. It involves 'active maintenance' of rules and goals within lateral areas of prefrontal cortex in a preventive fashion to facilitate future performance. In contrast, reactive control is less effortful mode and it involves allocating attention to rules and goals on an 'as needed' basis, once a problem (such as the occurrence of conflict or an error) has arisen.

Therefore, non-anxious individuals are found to alternate flexibly between reactive and proactive control modes in accordance with changing task demands. In contrast, anxious individuals may get distracted by worries that deplete resources needed for active goal maintenance, thereby interfering with proactive control and throwing anxious individuals into a reactive control mode i.e., anxious individuals rely more heavily on reactive control (Braver, 2012).

Again, effect of anxiety on an individual may vary depending on its interaction with the task performance, e.g., low to moderate anxiety is often described as beneficial as it enables the body to discharge energy equivalent to the task at hand. High anxiety, on the other hand, may be devastating because it excites the body system above normal functioning capacity and impacts negatively on task performance (Castello, 1976).

Behavioral and neuroimaging studies have also shown that anxiety can have an adverse effect on cognitive performance, especially on tasks that require attentional focus. In order to explain the attentional control in anxiety and cognitive performance, Eysenck proposed the attentional control theory (Eysenck et al., 2007). The theory predicts that anxiety can have a greater impact on performance efficiency of tasks requiring the shifting function (shifting back forth between tasks) and inhibition function (ability to deliberately inhibit dominant, automatic responses when necessary) of working memory (Miyake et al., 2000).

Also, anxiety has been found linked with attentional bias and with emotional interference while performing on Stroop task (MacLeod, 1991). The Stroop color-word interference task is one of the most widely used experimental tasks in cognitive psychology which is used to measure this interference as well as to measure why people cannot ignore some information that is irrelevant to their task goals and how irrelevant information affects performance (Stroop, 1935; MacLeod, 1991).

Stroop interference effect refers to the increase in response latency when an individual is required to identify the color of the color-word when these aspects of the stimulus are incongruent (word red presented in color blue) compared to the time required to name the color of congruent stimulus (word red presented in color red). 
Finally, studies on Stroop interference and anxiety reveal that anxious individuals show greater Stroop interference, and under emotional Stroop, anxious individuals are found to show greater interference for emotionally threatening words than for positive and neutral words (MacLeod, 1991).

Given the worry of teachers and parents nowadays with regard to the cognitive task performance linked anxiety of adolescents, the authors studied the cognitive performance of adolescents with and without emotional valence. For this purpose, it was felt that the subjects be categorized into low, moderate and high anxiety groups so that specific derivations can be made. This, in turn, would have implications for facilitating the understanding of and prevention of damage to individuals’ mental health as well as their performance.

\section{METHODOLOGY}

SAMPLE

A mixed sample of 89 students (43 boys \& 46 girls) was randomly chosen from the Government schools of Chandigarh. The age range was $14-18$ years (mean age $=15.9$ years).

\section{MEASURES USED}

A. IPAT Anxiety scale questionnaire (Cattell \& Scheier, 1963). The questionnaire consists of 40 items and the subjects has to respond in 'Yes', 'No' or 'Sometimes'.

B. The Stroop task (Stroop, 1935) :- The task measures the inhibition and interference facet of executive control. It consisted of two conditions :-

i. Cognitive Stroop task (b1) :- In this condition, the subjects are presented with color nouns and are asked to name the color of the ink in which the noun is written. Errors are counted for scoring purposes. An error occurs in case the subject names the color wrongly.

ii. Emotional Stroop task (b2) :- It is a version of the classic Stroop task wherein the subjects are presented with nouns which are emotionally valenced i.e. representing different emotions through nouns written in colors. The subject is asked to name the color of the noun while ignoring the semantic meaning of the noun.

\section{DESIGN}

A 3x2 repeated measures ANOVA design involving 3 levels of anxiety (low, moderate and high) and 2 levels of Stroop task (cognitive and emotional) with repeated measures on second factor was used.

\section{PROCEDURE}

The study comprised of two phases:

Phase 1 : In this phase, the IPAT Anxiety scale questionnaire by Cattell and Scheier (1963) was administered individually to all the subjects. 
The scoring was carried out as per the manual and the subjects were divided into three groups viz. Low anxiety (a1), Moderate anxiety (a2) and High anxiety (a3) groups as per the formula of Mean $\pm 1 / 2$ SD.

The classification may be seen in Table no.1 \& 2 .

\begin{tabular}{|l|l|}
\hline Mean $=37.30$ & S.D $=7.36$ \\
\hline
\end{tabular}

Table no. 1 :- Mean and SD of anxiety scores of the initial sample.

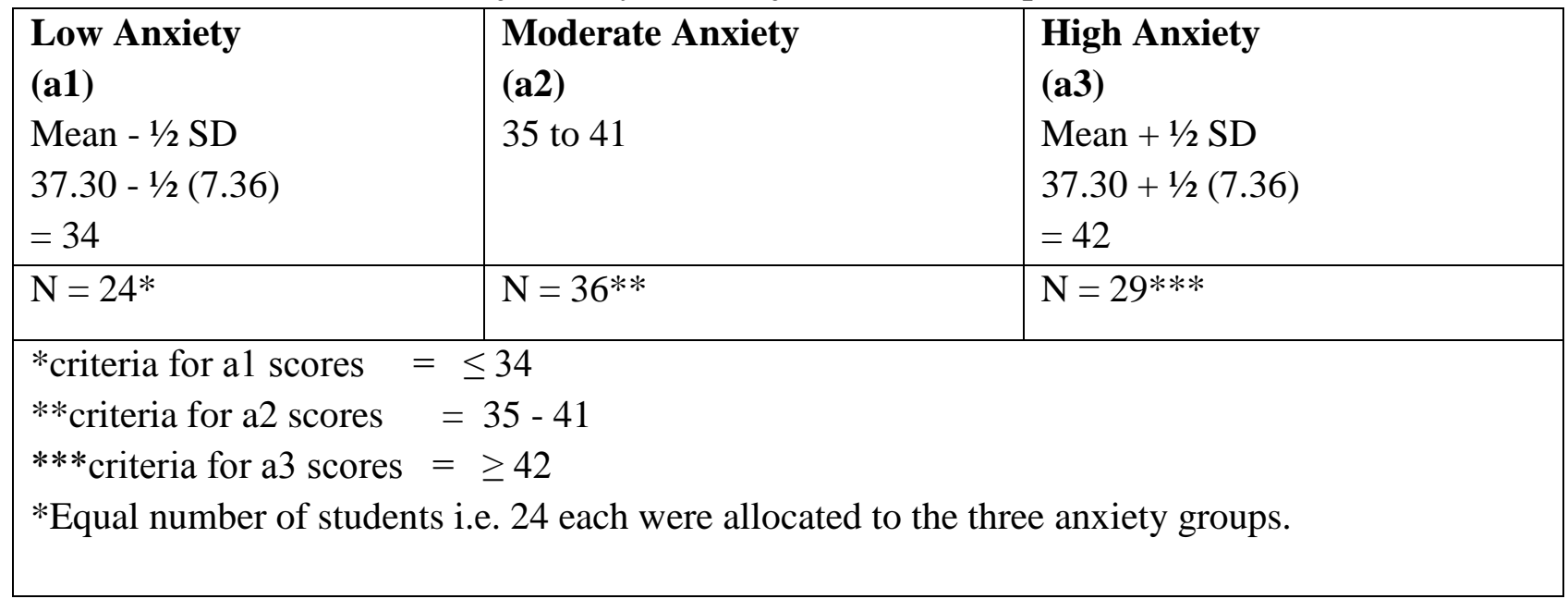

Table no. 2 :- Distribution of initial sample among the three groups. *

Phase 2 : The following Stoop experiment was conducted individually on low, moderate and high anxiety group subjects:

The Stroop experiment consisted of 2 levels i.e. cognitive (b1) and emotional (b2). A total of 5 trials were given to each subject. In each trial, there were 24 sub trials. Each sub trial comprised of 12 cognitive Stroop items and 12 emotional Stroop items. Each word was presented for 1.25 seconds. Stroop color words were individually presented. The error responses were noted down as a measure of actual performance.

After each trial, a blank screen was shown for 20 seconds to avoid fatigue in the subject. Precise instructions were given to the subjects and in order to avoid errors of habituation and anticipation. Proper randomization of conditions was adhered to.

The participating subjects were informed about the purpose and potential benefits of the study. Subsequently, the consent was taken from the subjects. In line with ethics policies, confidentiality of responses and privacy of the subjects was ensured 


\section{RESULTS AND DISCUSSION}

The aim of the present work was to study the effect of anxiety on task performance under cognitive and emotional Stroop conditions respectively. The results of the study are as under :-

\begin{tabular}{lcccc}
\hline Source of variation & SS & df & MS (SS/df) & F \\
\hline Between Ss & $\mathbf{2 0 0 2 3 . 9 1}$ & $\mathbf{7 1}$ & & \\
A (Anxiety) & 23701.14 & 2 & 11850.71 & $222.38^{* *}$ \\
SS.W. groups & 3677.23 & 69 & 53.29 & \\
(error between) & & & & \\
Within Ss & $\mathbf{7 4 9 5 . 8}$ & $\mathbf{7 2}$ & & \\
B (Task levels) & 18408.56 & 1 & 18408.56 & $160.55^{* *}$ \\
AB & 18824.43 & 2 & 9412.22 & $82.09^{* *}$ \\
B x SS.W. groups & 7911.67 & 69 & 114.66 & \\
(Error within) & & & & \\
Total & 27519.71 & 143 & & \\
**F (significant at .01 level) & & &
\end{tabular}

Table 3 :- Summary table of ANOVA for 3x2 (Repeated measures) factorial experiment.

As may be seen from above (see Table no. 3), anxiety has had a significant effect on the task performance with $\mathrm{F}(2,69)=222.38^{* *}, \mathrm{p}<0.01$. The task levels i.e., cognitive Stroop and emotional Stroop too were found to influence the performance significantly $F(1,69)=$ $160.55^{* *}, \mathrm{p}<0.01$. Further, significant interaction was found between anxiety and Stroop task conditions $\mathrm{F}(2,69)=82.09 * *$.

Maximum errors were seen in case of b2 of the high anxiety subjects (a3) followed by moderate anxiety subjects (a2) followed by low anxiety group subjects (a1) ( See Table no. 4). Again, a similar trend was seen in case of performance of a1, a2 and a3 on b1. That the a1 group was successfully able to fend off attention reorientation vulnerability under both the task conditions seems to indicate that if individuals can keep a tab on their anxiety level and somehow maintain their anxiety at a lower level, cognitive output would be better.

\begin{tabular}{|l|l|l|l|}
\hline Group & Variable & Mean (Errors) & \multirow{2}{*}{ Sig (two tailed) } \\
\hline \multirow{2}{*}{ Low Anxiety (a1) } & Cognitive Stroop errors (b1) & 6.33 & \multirow{2}{*}{.231} \\
\cline { 2 - 3 } & Emotional Stroop errors (b2) & 4.70 & \multirow{2}{*}{.822} \\
\hline \multirow{2}{*}{ Moderate anxiety (a2) } & Cognitive Stroop errors (b1) & 9.13 & \multirow{2}{*}{$.000^{* *}$} \\
\cline { 2 - 4 } & Emotional Stroop errors (b2) & 8.21 & \\
\hline \multirow{2}{*}{ High Anxiety (a3) } & Cognitive Stroop errors (b1) & 16.92 & \\
\cline { 2 - 4 } & Emotional Stroop errors (b2) & 22.50 & \\
\hline
\end{tabular}

${ }^{* *} \mathrm{p} \leq .01$, significant at .01 level.

Table no. 4 :- Mean and t-ratio results for cognitive and emotional Stroop errors for anxi.ety groups. 


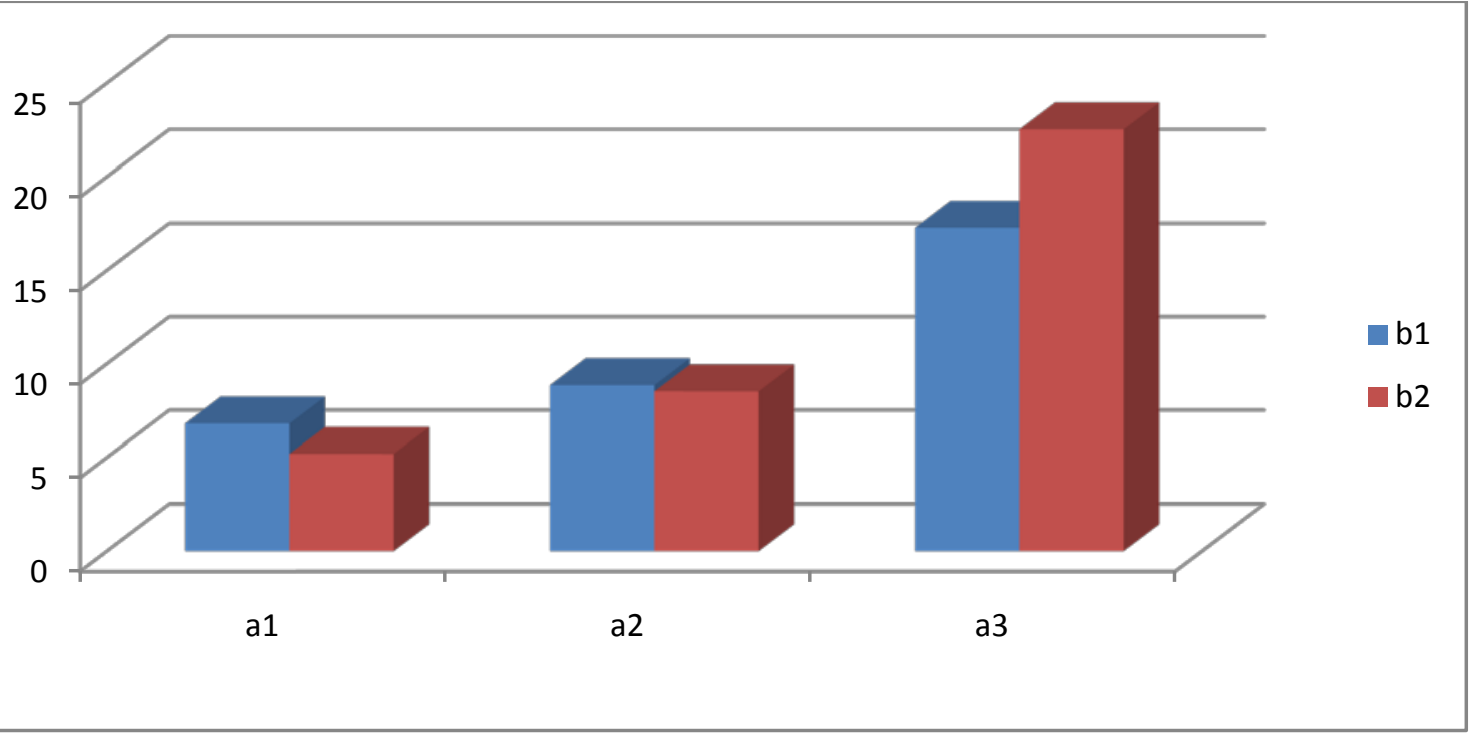

Figure 1 :- Trends for means of cognitive and emotional Stroop errors across the three anxiety groups.

In line with the above, Dresler (2009) reported that anxiety may enhance emotional interference with performance. Anxiety selectively facilitates early processing of threat and thus enhances distractibility to task-irrelevant stimuli. Attentional bias for threat-related material (Bar-Haim et al., 2007) thus assumes importance in such cases.

t- test results reveal significant differences in scores on b1 and b2 of a3 level subjects ( see Table no. 4) and perhaps indicate the inability of the a3 group to yield better performance on b2. As may be seen, the errors on b2 are almost five times more than those of low anxiety group subjects.

The results seem to echo the finding of Owens et al (2012) that anxiety can have a negative effect on working memory performance. That this leads to emotional interference while performing on Stroop task has been indicated in many studies (e.g. MacLeod, 1991).

Emotional intensity of stimulus nouns interferes with the ongoing cognitive task. Only those linguistic items which have intense affective charge have been reported to be salient enough to reorient attention in a bottom-up fashion, thereby disrupting the ongoing task (Carretie et al., 2008).

The present research findings seem to be in line with findings that high levels of anxiety are often harmful, but low levels can be helpful (e.g. Grills-Taquechel, 2012). Therefore, anxiety just might not be a maladaptive state which needs to be removed in order to perform optimally (Jackson \& Csikszentmihalyi 1999) and a moderate amount of anxiety seems to enhance performance when an individual's skill level matches the performance demands of the situation 
and the individual interprets that anxiety positively (Papageorgi et al., 2013). High anxiety individuals seem to be performing through reactive control because they seem to be distracted by emotional tone in the emotional Stroop and thus shown low performance on the latter. In the present study, the low anxiety and moderate anxiety individuals seem to be using more proactive control and seem to be low on reactive control (Braver, 2012) because their emotional Stroop performance is better than their cognitive Stroop performance but perhaps due to the effect of anxiety, their performance gets lowered.

Interestingly, it has been reported that the typical "fight or flight" response to anxiety tends to motivate success oriented students to "fight", that is, to approach a performance situation and undertake the necessary preparation required to achieve optimal performance outcomes (Martin and Marsh., 2008).

The present study seems to indicate that certain amount of anxiety may be required as a drive towards positive action and an excess of the same could be detrimental to task performance and this happens due to attention reorientation because of emotional pressure on working memory attending the cognitive output of the students. Such an impact of emotional nouns highlights the emotional vulnerability of individuals resulting in cognitive resource wastage and fragile emotional stability during times of cognitive performance. The presence or absence of such emotional fragility assumes importance in the mental health as well as cognition linked performance of adolescent students, as indicated earlier.

\section{REFERENCES}

Bar-Haim, Y., Lamy D., Pergamin L., Bakermans-Kranenburg M. J., Van Ijzendoorn M. H. (2007). Threat-related attentional bias in anxious and nonanxious individuals: a metaanalytic study. Psychological Bulletin, 133(1), 1-24.

Braver, T. S. (2012). The variable nature of cognitive control: a dual mechanisms framework. Trends Cogn. Sci, 16, 106-113.

Carretie, L., Hinojosa, J. A., Albert. J, Sara, P., Belen, S., Jose, I., Mari, S. (2008). Psychophysiology, 45, 188-196.

Castello, C. G. (1976). Anxiety and depression: The adaptive emotions. McGall-Queen's University Press. Montreal.

Cattell, R.B., \& Scheier, I. H. (1963). Handbook for the IPAT anxiety scale questionnaire. Institute for Personality and Ability Testing. Champaign IL. Institute for Personality and Ability Testing.

Dresler, T., Mériau, K., Heekeren, H.R., Meer, E.V. (2009). Psychological Research, 73, 364371. DOI 10.1007/s00426-008-0154-6 123.

Eysenck, M. W., Derakshan, N., Santos, R., \& Calvo, M. G. (2007). Anxiety and cognitive performance: The processing efficiency theory. Emotion, 7(2), 336-353.

Grills-Taquechel, A. E., \& Ollendick, T. H. (2012). Phobic and anxiety disorders in children and adolescents. Boston: Hogrefe Publishers.

Huberty, T. J. (2009). Test and performance anxiety. Principal Leadership, 10, 12-16. Retrieved from http://www.nasponline.org/on 24th February, 2014.

Jackson, S.A, \& Csikszentmihalyi, M. (1999). Flow in Sport. Human Kinetics, Champaign, IL. 
MacLeod,C.M.(1991). Half a century of research on the Stroop effect: An Integrative review. Psychological Bulletin, 109, 163-203.doi:10.1037/0033-2909.109.2.163.

Management of mental disorders (Vol. 1, Treatment Protocol Project). (2004). World Health Organisation Collaborating Centre for Evidence in Mental Health Policy, Darlinghurst, NSW.

Martin, A.J., \& Marsh, H.W. (2008). Academic buoyancy: towards an understanding of students' everyday academic resilience. Journal of School Psychology, 46(1), 53-83.

Miyake, A., Friedman, N. P., Emerson, M. J., Witzki, A. H., Howerter, A., \& Wager, T. D. (2000). The unity and diversity of executive functions and their contributions to complex “frontal lobe” tasks: A latent variable analysis. Cognitive Psychology, 41(1), 49-100.

Nadeem, M., Ali, A., Maqbool, S., \& Zaidi, S.U. (2012). Impact of anxiety on the academic achievement of students having different mental abilities at university levels in Bhawalpur (Southern Punjab) Pakistan. International Online Journal of Educational Sciences, 4(3), 519-528.

Öhman, A. (2000). "Fear and anxiety: Evolutionary, cognitive, and clinical perspectives". In Lewis, Michael; Haviland-Jones, Jeannette M. Handbook of emotions. New York: The Guilford Press. pp. 573-93. ISBN 978-1-57230-529-8.

Owens, M., Stevenson, J., Hadwin, J. A., \& Norgate, R. (2012). Anxiety and depression in academic performance: An exploration of the mediating factors of worry and working memory. School Psychology International, 33(4), 433-449.

Papageorgi, I, Creech, A, Welch, G. (2013). Perceived performance anxiety in advanced musicians specializing in different musical genres. Psychology of Music, 41(1), 18-41.

Stroop, J.R. (1935). Studies of interference in serial verbal reactions. Journal of Experimental Psychology, 18(6), 643-662. 